\title{
Matrix Characterization of Generalized Hamming Weights
}

\author{
G. Viswanath \\ Dept. of Elect. Comm. Engg. \\ Indian Institute of Science \\ Bangalore 560012, India \\ e-mail: gviswa@protocol. \\ ece.iisc.ernet.in
}

B. Sundar Rajan ${ }^{1}$

Dept. of Elect. Comm. Engg.

Indian Institute of Science

Bangalore 560012, India

e-mail: bsrajan@ece.iisc.

ernet.in

- For $i=1,2, \ldots, \mu$

1. and $i<g \leq \min \left\{d_{i}-1, k\right\}$, every $(g-2 i+1+\mu, g)$ sub matrix has rank $\geq(g-i+1)$.

2. there exist a $g, i<g \leq \min \left\{d_{i}, k\right\}$, such that $(g-2 i+\mu, g)$ sub matrix has rank equal to $(g-i)$.

- For $i=(\mu+1), \ldots, k$

1. For $1<g \leq \min \{(n-k),(k-\mu)\}$ every $(g, g+\mu)$ sub matrix has rank $g$.

Corollary 2: ( $N^{2} M D S$ code) For $N^{2} M D S$ codes the systematic generator matrix characterization is

1. For $1<g \leq \min \{(n-k-2), k\}$ every $(g+2, g)$ sub matrix has rank $\geq \mathrm{g}$

The support of a linear code $C$ is the set of coordinate positions, where not all codewords of $C$ are zero. The $r$-th generalized Hamming weight $d_{r}, 1 \leq r \leq k$, of a $[n, k]$ linear code $C$ over a field is defined as the cardinality of the minimal support of an $[n, r]$ subcode of $C$. The sequence $\left(d_{1}, d_{2}, \cdots, d_{k}\right)$ is called the Hamming Weight Hierarchy (HWH) of $C$ [3]. The relation $d_{r}=n-k+r$ for $r=1,2, \cdots, k$ characterizes MDS codes.

NMDS Codes: [1] The class of $[n, k]$ codes where $d_{1}(C)=$ $(n-k)$, and $d_{i}(C)$ is $(n-k+i)$ for $i=2,3, \ldots, k$.

$N^{2} M D S$ Codes: [2] The class of $[n, k]$ codes where $d_{1}(C)=$ $(n-k-1), d_{2}(C)=(n-k+1)$ and $d_{i}(C)$ is $(n-k+i)$ for $i=3,4, \ldots, k$.

We generalize NMDS and $N^{2} M D S$ codes as $N^{\mu} M D S$ Codes: The class of $[n, k]$ codes where $d_{i}(C)=$ $(n-k+2 i-\mu-1)$ for $i=1,2, \ldots, k$.

\section{Matrix Characterization of HWH}

The $M D S$ discrepancy of an $[n, k]$ code is defined as the smallest $\mu$ such that $d_{\mu+1}=n-k+\mu+1$.

Theorem 1: The systematic parity check matrix of an $\left[\begin{array}{ll}n k & k\end{array}\right]$ linear code with $M D S$ discrepancy $\mu$ and $d_{i}(C)=n-k+i-\eta_{i}$ can be characterized as follows:

- For $i<g \leq \min \left\{d_{i}-1, k\right\}$, every $\left(g+\eta_{i}+1-i, g\right)$ sub matrix of $P$ has rank $\geq(g-i+1)$.

- There exists a $g, i<g \leq \min \left\{d_{i}, k\right\}$, such that rank of $\left(g-i+\eta_{i}, g\right)$ sub matrix is $(g-i)$

- For $1<g \leq \min \{(n-k),(k-\mu)\}$ every $(g, g+\mu)$ sub matrix has rank $g$.

For $N^{\mu} M D S$ codes the defect, $\eta_{i}$, for $1 \leq i \leq \mu$, is $(\mu+1-i)$. The defect is zero for all $i>\mu$.

Corollary 1: ( $N^{\mu} M D S$ codes) The systematic $G$ matrix characterization of $N^{\mu} M D S$ codes is as follows:

\footnotetext{
${ }^{1}$ This work was partly supported by DST, India, through Research Grant No:III.5(31)/99-ET to B. S. Rajan
}

2. For $2<g \leq \min \{(n-k), k\}$ every $(g, g)$ sub matrix has rank $\geq(g-1)$

3. For $1<g \leq \min \{(n-k-1), k\}$ there exits a $(g+1, g)$ sub matrix with rank $(g-1)$.

4. For $2<g \leq \min \{(n-k+1), k\}$ there exits a $(g-1, g)$ sub matrix with rank $(g-2)$.

5. For $1<g \leq \min \{(n-k),(k-2)\}$ every $(g, g+2)$ sub matrix has rank $g$.

Corollary 2.1: For $k>q>3$ and $n>2 q-1+k$ the systematic generator matrix of a $N^{2} M D S$ code is characterized by every $(g+2, g)$ submatrix having rank $\geq g$.

Corollary 3: ( $N M D S$ code) Systematic generator matrix characterization of $N M D S$ codes is:

- For $1<g \leq \min \{(n-k-1), k\}$ every $(g+1, g) \mathrm{sub}$ matrix has rank $\geq \mathrm{g}$

- For $1<g \leq \min \{(n-k), k\}$ there exists a $(g, g)$ sub matrix with rank equal to $(g-1)$

- For $1<g \leq \min \{(n-k),(k-1)\}$ every $(g, g+1)$ sub matrix has rank $g$.

Corollary 3.1: If $n>(k+q)$ the systematic generator matrix of a $N M D S$ code is characterized by every $(g+1, g)$ submatrix having rank $\geq \mathrm{g}$.

\section{REFERENCES}

[1] S. D. Dodunekov and I. N. Landgev, ' On Near-MDS Codes', Technical Report, No:Lath-ISY-R-1563, Department of Electrical Engineering, Linkoping University, February, 1994.

[2] J. Olsson, ' On Near-Near-MDS Codes', Proceedings of Algebraic and Combinatorial Coding Theory Workshop, June, 1996

[3] V. K. Wei, "Generalized Hamming Weights for linear codes," IEEE Trans. on Information Theory, IT-Vol.37, No:5, Sept.1991, pp.1412-1418. 